\title{
Tunable pattern transitions in a liquid-crystal-monomer mixture using two-photon polymerization
}

\author{
Chandroth P. Jisha, ${ }^{1}$ Kuei-Chu Hsu, ${ }^{2}$ YuanYao Lin, ${ }^{1}$ Ja-Hon Lin, ${ }^{3}$ Chien-Chung Jeng, ${ }^{4}$ and Ray-Kuang Lee ${ }^{1, *}$ \\ ${ }^{1}$ Institute of Photonics Technologies, Frontier Research Center on Fundamental and Applied Sciences of Matters, \\ National Tsing-Hua University, Hsinchu 300, Taiwan \\ ${ }^{2}$ Unice E-O Services Inc., Chung-Li 320, Taiwan \\ ${ }^{3}$ Department of Electro-Optical Engineering and Institute of Electro-Optical Engineering, \\ National Taipei University of Technology, Taipei 106, Taiwan \\ ${ }^{4}$ Department of Physics, National Chung-Hsing University, Taichung 402, Taiwan \\ *Corresponding author: rklee@ee.nthu.edu.tw \\ Received August 6, 2012; revised October 16, 2012; accepted October 31, 2012; \\ posted November 1, 2012 (Doc. ID 173832); published November 28, 2012

\begin{abstract}
Through two-photon lithographic processes, we report experimentally and numerically a series of photoinduced tunable polymerization patterns in shapes from straight channel, serpentine curve, to periodic grating when an ultrashort femtosecond laser pulse directly writes in a liquid-crystal-monomer mixture along a line for different scanning speeds. Laser beams with polarization perpendicular to the direction of writing and the alignment of liquid crystals, produce snake-shaped patterns at an intermediate scan rate. (C) 2012 Optical Society of America
\end{abstract} \\ OCIS codes: $230.0230,160.3710,160.5335$.
}

Due to the flexibility in production process, direct writing technologies have the capability to produce a broad range of complex structures. For polymer-dispersed liquid crystals (PDLCs), compared to thermal, solvent, and electrical approaches, direct laser writing is demonstrated as a promising method to induce polymerization in micromachining and nanomachining [1] . Composited by spreading submicrometer-sized liquid crystal (LC) droplets in a polymer matrix, PDLCs have shown both electrical and optical tunabilities, and have been applied to a variety of applications in optical devices from light switches, phase modulators, holographic gratings, to flat panel displays. Depending on the profile of writing beam and interplay among photoinduced polymerization, phase separation morphologies, and diffusion mechanism of monomer molecules, different structures like gratings, waveguides, and broken stripes are demonstrated [2-5]. In comparison to one-photon absorption, in recent decades, two-photon (TP) lithography has emerged as a promising technique for direct laser writing, for its capacity to fabricate fine and well formed patterns, as well as three-dimensional structures, microscaled in size and nanoscaled in resolution [므, ]. Commercially available photopolymerizable monomers are generally transparent to infrared light and allow a deeper penetration, which makes them excellent candidates for two-photon polymerization (TPP). Although TP direct laser writing has been widely used for local alignment of LCs in predetermined structures like gratings, it has not been directly used to demonstrate transition of written patterns. Such transitional patterns have been observed in several hydrodynamic systems exhibiting symmetry-braking instabilities, resulting in patterns like coiling, buckling of jets, and meandering [ $\underline{8}-10]$.

In this work, by scanning the laser along a straight line, formation and transition of various structures in the form of straight waveguide, serpentine patterns, and microgratings are demonstrated experimentally, through the TPP lithography in PDLCs by varying only the scanning speed of the laser. The direction of polarization of the writing beam plays an important role in the formation of curved structures, for which an original sinusoidally modulated laser intensity is transferred into a curved serpentine shape. Optical images taken from parallel and perpendicular analyzers reveal the reorientation of LCs, and a theoretical model with the introduction of convection flow is developed to explain these observed symmetry-breaking structures. The experimental setup for the fabrication is illustrated in Fig. 1. The LC-monomer mixture consisting of $30 \mathrm{wt}$ \% of nematic LC $E 7$ [11,12], 69.4 wt. \% monomer NOA65 (Norland), 0.5 wt. \% photoinitiator Rose bengal (Oregon medical laser center), and 0.1 wt. \% of dye bis (2,4,6-trimethylbenzoy) phenylphosphine oxide (Ciba: Irgacure 819) is sandwiched between two glass slides with a separation of $30 \mu \mathrm{m}$. The glass surfaces are treated with DMOAP (N, N-dimethyl-Noctadecyl-3-aminopropyl-trimethoxysilyl chloride 6), in order to let the LC molecules stay in a hometropically

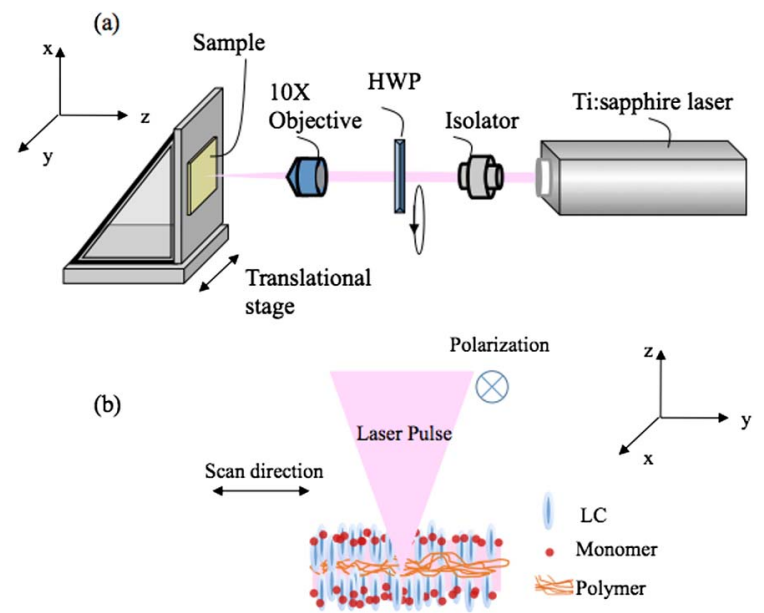

Fig. 1. (Color online) (a) Schematic of the experimental setup. A frequency-doubled Ti:sapphire laser is focused on the sample and performs the writing along the $y$ axis, where the half-wave plate (HWP) is used to control the polarization of input laser. (b) Illustration for the directions of laser polarization, laser scanning, and the orientation of LCs. 
aligned state (along the $z$ axis). The sample is mounted on a controllable three-axes translational stage, allowing us to fine tune its position. A commercial tunable femtosecond Ti:sapphire oscillator (Spectra-Physics Tsunami), with the wavelength at $720 \mathrm{~nm}$, laser pulse duration of $300 \mathrm{fs}$, repetition rate at $80 \mathrm{MHz}$, and power at $800 \mathrm{~mW}$, is focused on the sample by a $10 \times$ objective lens giving a spot diameter around $12 \mu \mathrm{m}$ at the focal point, along with a depth of focus $1.2 \mathrm{~mm}$. The sample is translated in a straight line (denoted as the $y$ axis). The peak intensity at the focus point is $8.6 \times 10^{15} \mathrm{~W} / \mathrm{cm}^{2}$, while the average intensity there is $7.0 \times 10^{5} \mathrm{~W} / \mathrm{cm}^{2}$.

Varying the scanning speed results in different patterns, as shown in Fig. 2. To verify any possible re-orientation of LCs, a pair of polarizer and analyzer is used to take the optical microscopic (OM) images. When the direction of polarizer and analyzer is parallel, as shown in Fig. 2(a), patterns generated by TPP at different scanning speeds are shown in Figs. 2(c)-2(f). In these images, the formed polymer structures are identified (through SEM images) as the bright region [5]. For a slower writing speed, $0.1 \mathrm{~mm} / \mathrm{s}$, a straight guide is formed as shown in Fig. 2(c); whereas for a faster writing speed, $2 \mathrm{~mm} / \mathrm{s}$, a micrograting structure is formed, Fig. 2(f). Upon irradiation by the laser, the material lying in the vicinity of the focal point cures under the process of TPP due to a higher photon density in that region. Together with the scanning beam, newly formed polymers as well as reorienting LCs result in several processes acting in tandem, namely, diffusion, convection, and reorientation. A balance of these processes results in the observed structures. The formation of straight guides at low scanning speeds and micrograting structure at high scanning speeds are fairly intuitive and expected. When the writing speed is slow, optical dose received at each scanning point along the translation direction is almost constant. The reaction time is longer in this case. As a result, a uniform straight guide with a

(a)
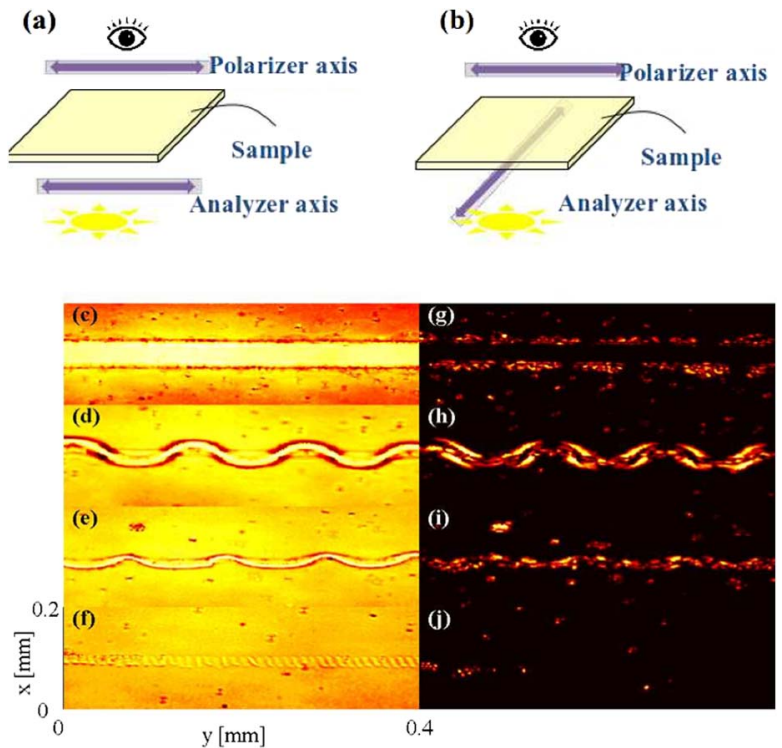

Fig. 2. (Color online) Optical microscopic images of the patterns formed in a LC-monomer mixture taken with the direction of analyzer (c)-(f) parallel and (g)-(j) perpendicular to the polarizer. The scan speeds for TP laser lithography are (c),(g) 0.1, (d),(h) 0.5 , (e),(i) 1, and (f),(j) $2 \mathrm{~mm} / \mathrm{s}$, respectively. high polymer concentration is formed along the scanning direction. When the scan speed is high enough, the optical dose is modulated along the writing direction resulting in a modulated pattern of alternate polymer-rich and polymer-scarce regions manifested as microgratings. To one's surprise, for an intermediate scan speed, a sinusoidal-like structure with a serpentine pattern is obtained, for scan speeds of $0.5 \mathrm{~mm} / \mathrm{s}$ and $1 \mathrm{~mm} / \mathrm{s}$, see Figs. 2(d) and 2(e). In this case, the increase in scan speed makes the optical dose as a sinusoidal modulation in the intensity distribution along the writing direction, which is insufficient to induce a uniform polymerization. The resulting serpentine pattern is an asymmetric profile along the writing direction. We want to emphasize that such patterns are strongly dependent on the polarization of writing beam. When TPP is performed with the polarization of writing field parallel to the scanning direction ( $y$ axis), the sinusoidal patterns and gratings vanish and the results are essentially equivalent to that found for one-photon polymerization [5], indicating that processes resulting from LC reorientation may play a role in the formation of sinusoidal patterns. In the configuration where the direction of the analyzer is rotated by $90^{\circ}$, Fig. 2(b), one can clearly see that the bright regions reflect the spatial distribution of reoriented LC molecules that have been pushed to the sides of polymer structures, as shown in Figs. $2(\mathrm{~g})-2(\mathrm{j})$. The LC molecules in the polymer-rich area are not reoriented as they are trapped along with the cross-linked polymers.

To understand the observed patterns, we propose a phenomenological two-dimensional diffusion model by accounting for convection in the sample resulting from the local perturbations due to the reorientation of LC molecules in the vicinity of laser scanning. Let $\phi_{m}, \phi_{p}$, and $\phi_{\mathrm{lc}}$ be the molar concentrations of monomer, polymer, and LC, respectively. Using nondimensional units $x=x^{\prime} / w_{0}, y=y^{\prime} / w_{0}$, and $t=t^{\prime} /\left(k_{p} I_{0}\right)$, with $w_{0}$ the width of writing beam $(50 \mu \mathrm{m}), I_{0}$ its intensity, and $k_{p}$ the polymerization rate, the rate at which the concentration of monomer molecules change can be written according to a modified Fick's law []]

$$
\frac{\partial \phi_{m}}{\partial t}=\left[\nabla(D(x, y, t) \nabla)-F(x, y, t)+I\left(1-\phi_{m}^{2}\right)\right] \phi_{m},
$$

where the first term accounts for the diffusion of monomers with diffusion coefficient given by $D(x, y, t)=$ $D_{0} \exp \left[-\alpha \phi_{p}(x, y, t)\right]$, the second term represents decrease in concentration due to polymerization with the local polymerization rate given by $F(x, y, t)=$ $K_{0} \exp \left[-\alpha \phi_{p}(x, y, t)\right] I(x, y), \alpha$ is related to the weight of the monomer molecules and $I=A_{0} \exp \left(-x^{2} / w_{0}^{2}\right)$ $\cos ^{2}(f y)$ describes the intensity profile. The laser scan speed is controlled by the parameter $f$; increasing $f$ implies increasing scan speed. The last term is an additional force due to the writing beam acting on the monomer molecules with the TP process, which keeps the monomer in the bright region. Similarly, the reaction equation for change in concentration of polymer molecules can be written as

$$
\frac{\partial \phi_{p}}{\partial t}=\nabla\left(D(x, y, t) \nabla \phi_{p}\right)+u \nabla \phi_{p}+F(x, y, t) \phi_{m},
$$




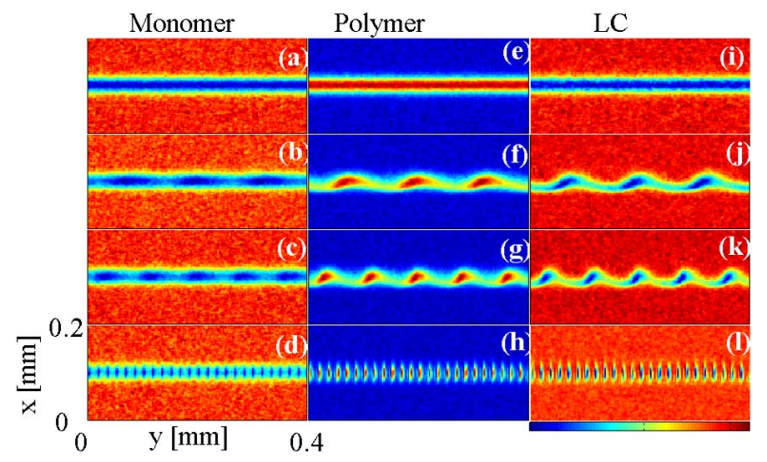

Fig. 3. (Color online) Simulation results for the concentrations of (a)-(d) monomer, (e)-(h) polymer, and (i)-(l) LC molecules are plotted in the columns respectively for laser scan parameters $f=0.01,2,3$, and 15 (from the top row to the bottom). Other parameters used are $|u|=25, D_{0}=0.1$, $K_{0}=1, \alpha=0.7$, and $A_{0}=1$, respectively.

with $\phi_{p}(x, y, 0)=0$ as the initial condition. The second term is a convection term and the flow velocity $u$ is proportional to the input laser profile $I$ as the flow is a geometrically asymmetrical after effect from the reorientation of LCs brought by the laser scan. Generally, the formed polymers are highly cross-linked and their diffusion can be neglected. But here we assume that the polymer molecules can also undergo diffusion and include the effect in the simulation. We do not make any attempt to find the number of LC molecules that have been reoriented, but qualitatively follow the fact that the polarization of the laser affects the reorientation of LC molecules. When the laser is polarized perpendicular to the writing direction it reorients the LC molecules whereas when it is polarized parallel to the writing direction no reorientation of LC takes place. Reorienting LC molecules induce a local flow velocity, and the effect of which is included in the equation for polymer concentration. The law of total volume conservation $\phi_{\mathrm{lc}}+\phi_{m}+\phi_{p}=1$ can be used to calculate the concentration profile of LC. Figure 3 shows the typical simulation results. The columns plot the monomer, polymer, and LC concentrations, respectively. It can be clearly seen that as $f$ increases (along the rows) there is a distinct pattern transition from guide-like, sinusoidal, and finally to micrograting structures. A dimensionless parameter, $R=D_{0} f^{2} /\left(K_{0} A_{0}\right)$ can be defined variation of which produces the different patterns. For a slow scanning speed (first row), the polymer profile is in the form of a straight guide as observed in OM images. Optical dose is received in a quasistationary manner so that the reorientation of LC does not result in a local perturbation. For intermediate scan speeds, the effect of flow velocity is evident and the pattern takes the form of a sinusoidal structure (second and third row). For a high scan speed, a micrograting structure (bottom row) is formed as expected. From the figures we can clearly see that the theoretical model is in very good agreement with the experimental observations.

In conclusion, using TP femtosecond direct laser writing process, at different scan speeds, on the LC-monomer mixture, we demonstrated and modeled the formation of straight guides, microgratings, and serpentine structures. Our results provide a step to control the fabrication of functional optical devices as well as to understand the complex effects taking place in hydrodynamic systems.

\section{References}

1. D. K. Yang and S. T. Wu, Fundamental of Liquid Crystal Devices (Wiley, 2006).

2. C. C. Bowley and G. P. Crawford, Appl. Phys. Lett. 76, 2235 (2000).

3. R. Sigel, G. Fytas, N. Vainos, S. Pispas, and N. Hadjichristidis, Science 297, 67 (2000).

4. A. Hoischen, S. A. Benning, and H.-S. Kitzerow, Appl. Phys. Lett. 93, 131903 (2008).

5. C. P. Jisha, K.-C. Hsu, Y. Y. Lin, J.-H. Lin, K.-P. Chuang, C.-Y. Tai, and R.-K. Lee, Opt. Mater. Express 1, 1494 (2011).

6. Y. Chou, P. R. Krauss, and P. J. Renstrom, Science 272, 85 (1996).

7. S. Kawata, H.-B. Sun, T. Tanaka, and K. Takada, Nature 412, 697 (2001).

8. C. Dombrowski, B. Lewellyn, A. I. Pesci, J. M. Restrepo, J. O. Kessler, and R. E. Goldstein, Phys. Rev. Lett. 95, 184501 (2005).

9. A. I. Pesci, M. A. Porter, and R. E. Goldstein, Phys. Rev. E 68, 056305 (2003).

10. N. Le Grand-Piteira, A. Daerr, and L. Limat, Phys. Rev. Lett. 96, 252503 (2006).

11. S.-T. Wu, E. Ramos, and U. Finkenzeller, J. Appl. Phys. 68, 78 (1990).

12. S.-T. Wu, Phys. Rev. A 33, 1270 (1986). 